\title{
Superpartner Solutions of a BPS Monopole in Noncommutative Space
}

\author{
Euy Soo $\mathrm{Na}^{\text {目 }}$ \\ Department of Physics \\ and Basic Sciences Research Institute, \\ Kyunghee University, Seoul, 130-701, Korea
}

\begin{abstract}
We construct $U(2)$ BPS monopole superpartner solutions in $N=2$ non-commutative super Yang-Mills theory. Calculation to the second order in the noncommutative parameter $\theta$ shows that there is no electric quadrupole moment that is expected from the magnetic dipole structure of noncommutative $U(2)$ monopole. This might give an example of the nature of how supersymmetry works not changing between the commutative and noncommutative theories.
\end{abstract}

\footnotetext{
1 esna@theory.khu.ac.kr
} 
The decoupling limit of the world volume theory on D3-branes in the NS-NS 2-form background is described by the noncommutative super Yang-Mills theory, in which BPS monopoles exist as a stable state because it can be higgsed just like the ordinary SYM. The solution to the BPS equation of the $U(2)$ noncommutative monopole to the first order in $\theta$ has been studied in [1] [2], and the second order solution is in [3]. The solution has the generalized rotational invariance and exhibits a dipole structure [4] [5] [in the magnetic field of the monopold2.

On the other hand, it is well known that ordinary BPS monopoles of $N=2$ YangMills theory are invariant under half the supersymmetry generators and hence form a 4 -dimensional, short representation of the supersymmetry algebra $[7]^{3}$. From the work of Jackiw and Rebbi [9], we know that the angular momentum of spinning monopoles is carried by the quantized states of fermionic zero-modes. For a single BPS monopole, the fermionic zero-modes are generated by infinitesimal broken supersymmetry transformations. What we get by acting with a finite transformation is then the backreaction of the fermionic zero-modes on the other fields. Because of the quantized nature of the fermionic zero-mode states [9], the fields of the monopole superpartner solution are necessarily operator valued.

In [10] we have studied the long-range fields of the different states in the ordinary $N=2$ BPS monopole supermultiplet. Following the work of Aichelburg and Embacher on $N=2$ BPS black holes [11], we generate the fields of a monopole "superpartner" solution by acting on the bosonic monopole with an arbitrary, finite, broken supersymmetry transformation. In which we have found that the operator valued electric dipole moment is proportional to the angular momentum operator with a gyroelectric ratio $g=2$ and the quadrupole moment tensor is found to vanish identically for all spin states.

This vanishing quadrupole moment tesor is in contrast with the result of [11] on $N=2$ black hole superpartners for which these variations are nonzero, which is one of the motivation of this paper together with the fact that $U(1)$ part of the magnetic field of the noncommutative $U(2)$ monopole exhibits a dipole structure. If noncommutativity produces magnetic dipole structure to $U(2)$ monopole, then one can expect the electric quadrupole

2 This dipole structure can be visualized from the brane picture as D-string stretched between parallel D3-branes. When a background $B$ field is turned on along the branes,the suspended D-string is tilted because the two endpoints carry opposite charges.

3 See e.g. [8] for a good review of this subject. 
moment [12] and it would be interesting to see if there exists the electric quadrupole moment that is not found in ordinary $S U(2)$ monopole [10]. However, our calculation gives a negative answer. Up to $O\left(\theta^{2}\right)$ in tree level, we show that there is no quadrupole moment. Presumably, this result has something to do with the nature of how supersymmetry works not changing between the commutative and noncommutative theories.

In the following, we will construct the BPS monopole superpartner solutions of the $N=2$ noncommutative super Yang-Mils theory, by applying the Seiberg-Witten map to all superpartner fields to the second order in $\theta$. As a check, up to $O\left(\theta^{2}\right)$ we showed explicitly that the angular momentum operator and the electric dipole moment are independent of noncommutativity.

We restrict ourselves to the case where the non-vanishing component of the noncommutative parameter is $\theta_{12}=-\theta_{21}=\theta$, excluding the effect of time noncommutativity. We shall take $U(2)$ as the gauge group because $S U(2)$ is not closed under the $\star$-product that is defined by

$$
(f * g)(x) \equiv f(x) g(x)+\frac{i}{2} \theta^{\rho \sigma} \partial_{\rho} f(x) \partial_{\sigma} g(x)-\frac{1}{8} \theta^{\rho \sigma} \theta^{\alpha \beta} \partial_{\rho} \partial_{\alpha} f(x) \partial_{\sigma} \partial_{\beta} g(x)+O\left(\theta^{3}\right)
$$

It replaces an ordinary multiplication in describing noncommutative theory. Small $\theta$ expansion is adopted to all fields. For example, the scalar Higgs field

$$
\hat{S}=\hat{S}^{A} T^{A}=\left(\hat{S}^{a}+\hat{S}_{(1)}^{a}+\hat{S}_{(2)}^{a}\right) T^{a}+\left(\hat{S}^{0}+\hat{S}_{(1)}^{0}+\hat{S}_{(2)}^{0}\right) T^{0},
$$

where the quantities with a hat denote those in the noncommutative description, the subscripts $(n)$ denote the quantitities at $O\left(\theta^{n}\right), a=1,2,3$, and $T^{A}$ are the anti-hermitian generators of $U(2)$ Lie algebra. Throughout this paper, this notation will be understood and other settings are the same as in [10].

We now turn to the construction of the noncommutative BPS monopole superpartner solutions $A$. We work in $N=2$ Yang-Mills theory with gauge group $U(2)$. The lagrangian is given by

$$
\begin{aligned}
\mathcal{L}_{N=2} & =\operatorname{Tr}\left(-\frac{1}{4} \hat{F}_{\mu \nu} * \hat{F}^{\mu \nu}-\frac{1}{4}\left(D_{\mu} \hat{P}\right)^{2}-\frac{1}{2}\left(D_{\mu} \hat{S}\right)^{2}-\frac{e^{2}}{2}[\hat{S}, \hat{P}]_{*}^{2}\right. \\
& \left.+i \hat{\bar{\psi}} \gamma^{\mu} * D_{\mu} \hat{\psi}-e \hat{\bar{\psi}} *[\hat{S}, \hat{\psi}]_{*}-e \hat{\bar{\psi}} \gamma_{5} *[\hat{P}, \hat{\psi}]_{*}\right),
\end{aligned}
$$

4 See [10] for ordinary superpartner solutions in detail. 
where all fields are $U(2)$ Lie algebra valued, e.g. $\hat{S}=\hat{S}^{A} \hat{T}^{A}, \hat{S}$ and $\hat{P}$ are two scalar Higgs fields and $\hat{\psi}$ is a Dirac fermion. The nonabelian electric and magnetic field strengths are defined by $\hat{E}^{A i}=-\hat{F}^{A 0 i}$ and $\hat{B}^{A i}=-\frac{1}{2} \epsilon^{i j k} \hat{F}_{j k}^{A}$.

Corresponding global supersymmetry transformations

$$
\begin{aligned}
\delta \hat{A}_{\mu} & =i \bar{\alpha} \gamma_{\mu} \hat{\psi}-i \hat{\bar{\psi}} \gamma_{\mu} \alpha, \quad \delta \hat{P}=\bar{\alpha} \gamma_{5} \hat{\psi}-\hat{\bar{\psi}} \gamma_{5} \alpha, \quad \delta \hat{S}=i \bar{\alpha} \hat{\psi}-i \hat{\bar{\psi}} \alpha \\
\delta \hat{\psi} & =\left(\frac{1}{2} \gamma^{\mu \nu} \hat{F}_{\mu \nu}-i \gamma^{\mu} D_{\mu} \hat{S}+\gamma^{\mu} D_{\mu} \hat{P} \gamma_{5}-i[\hat{P}, \hat{S}]_{*} \gamma_{5}\right) \alpha
\end{aligned}
$$

where the parameter $\alpha$ is a Grassmann valued Dirac spinor . For a static, BPS monopole field configuration with $\hat{P}=\hat{A}_{0}=\hat{\psi}=0$ and

$$
D_{i} \hat{S}^{A}=\frac{1}{2} \epsilon_{i j k} \hat{F}_{j k}^{A}
$$

only the fermion $\hat{\psi}$ has a nontrivial supersymmetry variation given by

$$
\begin{gathered}
\delta \hat{\psi}^{a}=-2 \gamma^{k}\left(D_{k} \hat{S}^{a}\right) P_{-} \alpha \\
\delta \hat{\psi}_{(1)}^{0}=-2 \gamma^{k}\left(D_{k} \hat{S}\right)_{(1)}^{0} P_{-} \alpha \\
\delta \hat{\psi}_{(2)}^{a}=-2 \gamma^{k}\left(D_{k} \hat{S}\right)_{(2)}^{a} P_{-} \alpha
\end{gathered}
$$

where $P_{ \pm}=\frac{1}{2}\left(1 \pm \Gamma_{5}\right)$ are projection operators with $\Gamma_{5}=-i \gamma_{0} \gamma_{5}$. If we define projected spinors $\alpha_{ \pm}$satisfying $P_{ \pm} \alpha_{ \pm}=\alpha_{ \pm}$, then $\alpha_{+}$generates unbroken supersymmetry transformations, while $\alpha_{-}$generates broken supersymmetry transformations. The variation $\delta \psi$ under a broken supersymmetry transformation gives a zero-mode of the fermion field equation in the monopole background.

At second order variations, $\delta^{2} S$ and $\delta^{2} A_{k}$ vanish and we find only nonzero variations for $P$ and $A_{0}$ given by

$$
\begin{gathered}
\delta^{2} A_{0}^{a}=-\delta^{2} P^{a}=-4 i\left(\alpha^{\dagger} \gamma^{k} \alpha\right) D_{k} S^{a}, \\
\left(\delta^{2} A\right)_{(1)}^{0}=-\left(\delta^{2} P\right)_{(1)}^{0}=-4 i\left(\alpha^{\dagger} \gamma^{k} \alpha\right)\left(D_{k} S\right)_{(1)}^{0}, \\
\left(\delta^{2} A_{0}\right)_{(2)}^{a}=-\left(\delta^{2} P\right)_{(2)}^{a}=-4 i\left(\alpha^{\dagger} \gamma^{k} \alpha\right)\left(D_{k} S\right)_{(2)}^{a} .
\end{gathered}
$$

These reduce to dipole fields in the long range limit. Interestingly, the third and fourth order variations of all the fields turn out to vanish even in the noncommutative sector. In

5 Our conventions for the Minkowski metric are "mostly minus" $\eta_{\mu \nu}=\operatorname{diag}(+1,-1,-1,-1)$ and $\gamma_{5}=+i \gamma_{0} \gamma_{1} \gamma_{2} \gamma_{3}$. 
particular, the third order variation of $\psi$ is found to be

$$
\begin{aligned}
\delta^{3} \psi^{a}= & 8 i\left(\alpha^{\dagger} \gamma^{k} \alpha\right)\left\{\gamma^{0} \gamma^{l} D_{l} D_{k} S^{a}+e \gamma^{0} \epsilon^{a b c}\left(D_{k} S^{b}\right) S^{c}\right\} P_{+} \alpha, \\
\delta^{3} \psi_{(1)}^{0}= & 8 i\left(\alpha^{\dagger} \gamma^{k} \alpha\right)\left\{\gamma^{0} \gamma^{l}\left\{\partial_{l} D_{k} S_{(1)}^{0}-\frac{1}{2} \theta^{\rho \sigma} \partial_{\rho}\left(D_{k} S^{a}\right) \partial_{\sigma} A_{l}^{a}\right\}\right. \\
& \left.\quad+e \gamma^{0} \frac{1}{2} \theta^{\rho \sigma} \partial_{\rho}\left(D_{k} S^{a}\right) \partial_{\sigma} S^{a}\right\} P_{+} \alpha, \\
\delta^{3} \psi_{(2)}^{a}=8 i\left(\alpha^{\dagger} \gamma^{k} \alpha\right)\left\{\gamma ^ { 0 } \gamma ^ { l } \left[\partial_{l} D_{k} S_{(2)}^{a}\right.\right. & -e \epsilon^{a b c}\left(\left(D_{k} S^{b}\right) A_{l(2)}^{c}-\frac{1}{8} \theta^{\rho \sigma} \theta^{\alpha \beta} \partial_{\rho} \partial_{\alpha}\left(D_{k} S^{b}\right) \partial_{\sigma} \partial_{\beta} A_{i}^{c}\right) \\
& \left.-\frac{1}{2} \theta^{\rho \sigma}\left(\partial_{\rho}\left(D_{k} S^{a}\right) \partial_{\sigma} A_{i(1)}^{0}+\partial_{\rho}\left(D_{k} S\right)_{(1)}^{0} \partial_{\sigma} A_{i}^{a}\right)\right] \\
+ & \gamma^{0}\left[e \epsilon^{a b c}\left(\left(D_{k} S^{b}\right) S_{(2)}^{c}+\left(D_{k} S\right)_{(2)}^{0}\right) S^{c}-\frac{1}{8} \theta^{\rho \sigma} \theta^{\alpha \beta} \partial_{\rho} \partial_{\alpha}\left(D_{k} S^{b}\right) \partial_{\sigma} \partial_{\beta} S^{c}\right) \\
- & \left.\left.\frac{1}{2} \theta^{\rho \sigma}\left(\partial_{\rho}\left(D_{k} S\right)_{(1)}^{0} \partial_{\sigma} S^{a}\right)\right]\right\} P_{+} \alpha,
\end{aligned}
$$

which vanish because $P_{+} \alpha=0$ for the broken supersymmetries. The fourth order variations of the bosonic fields then vanish because they are each proportional to $\delta^{3} \psi$. Note, the vanishing of the third and fourth order variations of the fields implies a vanishing quadrupole moment tensor for all states in the monopole BPS multiplet and is different from the variation of the $N=2$ black hole supermultiplet, for which these variations are nonzero [11]. It turns out that the BPS monopole exhibits no electromagnetic quadrupole structure in both the commutative and noncommutative spaces, and that the dipole structure of noncommutative monopole does not give rise to the electric quadrupole moment up to $O\left(\theta^{2}\right)$, the same of which holds apparently for any arbitrary higher order in $\theta$. This result is disappointing. It is mainly from the fact that noncommutativity influences only to the spacial part of field variations, not to the spin structure when supersymmetry transformation is done. In order to check this, let's see the invariance of the angular momentum operator.

The fermionic fields $\hat{\psi}^{A}$ may be expanded in the monopole background as

$$
\begin{aligned}
\hat{\psi}^{a \rho} & =-2\left(\gamma^{k}\right)^{\rho}{ }_{\sigma} \hat{\alpha}^{\sigma} D_{k} \hat{S}^{a}+\text { nonzero-modes } \\
\hat{\psi}^{0 \rho} & =-2\left(\gamma^{k}\right)_{\sigma}^{\rho} \hat{\alpha}^{\sigma}\left(D_{k} \hat{S}\right)_{(1)}^{0}+\text { nonzero-modes, } \\
\hat{\psi}_{(2)}^{a \rho} & \left.=-2\left(\gamma^{k}\right)^{\rho}{ }_{\sigma} \hat{\alpha}^{\sigma}\left(D_{k} \hat{S}\right)_{(2)}^{a}\right)+ \text { nonzero-modes }
\end{aligned}
$$

where $\rho, \sigma$ are spinor indices and we have explicitly displayed only the zeromode part of the expansion. Using the orthogonality of zero-modes and nonzero-modes, we can then 
express the spinorial parameters $\hat{\alpha}^{\lambda}$ and $\hat{\alpha}_{\lambda}^{\dagger}$ as ${ }^{\natural}$

$$
\hat{\alpha}^{\lambda}=+\frac{1}{2 M} \int d^{3} x\left(\gamma^{l}\right)_{\rho}^{\lambda} \hat{\psi}^{a \rho} D_{l} \hat{S}^{a}, \quad \hat{\alpha}_{\lambda}^{\dagger}=-\frac{1}{2 M} \int d^{3} x \hat{\psi}_{\rho}^{a \dagger}\left(\gamma^{l}\right)^{\rho}{ }_{\lambda} D_{l} \hat{S}^{a},
$$

where $M=4 \pi v / e$ is the mass of the monopolet and this form is that of the commutative case because the mass term arising from noncommutativity

$$
\int d^{3} x \eta^{k l}\left\{\left(D_{k} \hat{S}\right)_{(2)}^{a} D_{l} \hat{S}^{a}+\left(D_{k} \hat{S}^{a}\right) D_{l} \hat{S}_{(2)}^{a}+\left(D_{k} \hat{S}\right)_{(1)}^{0} \hat{S}_{(1)}^{0}\right\}=-M_{(2)}
$$

vanishes 8 . It is because the contributions from noncommutative fields fall off faster than $\frac{1}{r^{2}}$ compared to the commutative ones9, which makes the second order mass vanish. Consequently, the angular momentum vector has no correction from the noncommutativity as expected,

$$
J^{k}=2 i M\left(\alpha^{\dagger} \gamma^{k} \alpha\right)
$$

As an another check, we now turn to the long range limit of the electric field for the monopole superpartner solution up to $O\left(\theta^{2}\right)$. The result for the long range electric fields $\hat{E}^{i}=\hat{F}_{0 i} \equiv \frac{1}{v} \hat{S}^{A} * \hat{F}_{0 i}^{A}$ obtained are

$$
\begin{aligned}
E^{i} & =-\frac{2 i}{e}\left(\alpha^{\dagger} \gamma^{k} \alpha\right)\left\{\frac{3 x^{k} x^{i}}{r^{5}}-\frac{\delta^{k i}}{r^{3}}\right\}, \\
E_{(1)}^{i} & =0 \\
E_{(2)}^{i} & =-\frac{2 i}{e}\left(\alpha^{\dagger} \gamma^{k} \alpha\right)\{\text { no dipole field like terms }\},
\end{aligned}
$$

which shows that a dipole field with dipole moment vector $\vec{p}=-\frac{2 i}{e}\left(\alpha^{\dagger} \vec{\gamma} \alpha\right)$ can be seen only in commutative sector and that the electric dipole moment proportional to angular momentum operator, thus also the gyroelectric ratio $g=2$ 13 14 15, obtain no corrections from noncommutativity.

In conclusion, we considered $U(2)$ monopole in noncommutative space by constructing superpartner solutions up to $O\left(\theta^{2}\right)$. We found no electric quadrupole moment that is expected [12] by the dipole structure of noncommutative $U(2)$ monopole, which is because spin is indpendent of noncommutativity. As a check, up to $O\left(\theta^{2}\right)$ we showed explicitly

\footnotetext{
6 see [10] for angular momentum operator in detail

7 Here we have made use of the result $\int d^{3} x \eta^{k l}\left(D_{k} \hat{S}^{a}\right) D_{l} \hat{S}^{a}=-M$

$8 M_{(1)}$ also vanishes because the scalar solution is not influenced by noncommutativity at $O(\theta)$.

9 We use noncommutative BPS solutions in [1] [2] [3]
} 
that the angular momentum operator and the electric dipole moment obtain no correction from noncommutativity. In a more broad perspective, this result might give an example of the nature of how supersymmetry works not changing between the commutative and noncommutative theories.

Acknowledgements: We thank Dongsu Bak, David Kastor, Kimyeong Lee and Soonkeon Nam for helpful discussions and correspondence. This work is supported by BK21 Program of Korea Research Foundation. 


\section{References}

[1] K. Hashimoto, H. Hata and S. Moriyama, "Brane Configuration from Monopole Solution in Non-Commutative Super Yang-Mills Theory", JHEP 12 (1999) 021, hepth/9910196.

[2] D. Bak, "Deformed Nahm Equation and a Noncommutative BPS Monopole", Phys. Lett. B471 (1999) 149, hep-th/9910135.

[3] S. Goto and H. Hata, "Noncommutative Monopole at the Second Order in $\theta$ ", Phys. Rev. D62 (2000) 085022, hep-th/0005101.

[4] M. R. Douglas and M. Li, "D-Brane Realization of $N=2$ Super Yang-Mills Theory in Four Dimensions", hep-th/9604041.

[5] D. E. Diaconescu, "D-branes, Monopoles and Nahm Equations", Nucl. Phys. B503 (1997) 220, hep-th/9608163.

[6] A. Hashimoto and K. Hashimoto, "Monopoles and Dyons in Non-Commutative Geometry", JHEP (1999) 005, hep-th/9909202.

[7] E. Witten and D. Olive, "Supersymmetry Algebras That Include Topological Charges," Phys. Lett. 78B (1978) 97.

[8] J. Harvey, "Magnetic Monopoles, Duality and Supersymmetry", in Trieste HEP Cosmology 1995, hep-th/9603086.

[9] R. Jackiw and C. Rebbi, "Solitons With Fermion Number 1/2," Phys. Rev. D13 (1976) 3398.

[10] D. Kastor and E. S. Na, "Electric Dipole Moment of a BPS Monopole", Phys. Rev. D60 (1999) 025002, hep-th/9812191.

[11] P. C. Aichelburg, and F. Embacher, "Exact superpartners of $\mathrm{N}=2$ supergravity solitons," Phys. Rev. D34 (1986) 3006.

[12] I. Giannakis, J. T. Liu and M. Porrati, "Supersymmetric Sum Rules for Electromagnetic Multipoles", Phys. Rev. D58 (1998) 045016, hep-th/9803073.

[13] I. Giannakis and J. T. Liu, " $N=2$ Supersymmetry and Dipole Moments," Phys. Rev. D58 (1998) 2509, hep-th/9711173.

[14] H. Osborn, "Electric Dipole Moment for Supersymmetric Monopoles," Phys. Lett. 115B (1982) 226.

[15] S. Ferrara and M. Porrati, "Supersymetric Sum Rules on Magnetic Dipole Moments of Arbitrary Spin Particles," Phys. Lett. 288B (1992) 85. 